\title{
UNIVERSITYOF
}

FORWARD

THINKING

WESTMINSTER用

WestminsterResearch

http://www.westminster.ac.uk/westminsterresearch

\section{Day ahead hourly Price Forecast in ISO New England Market using Neuro-Fuzzy Systems}

Alshejari, A. and Kodogiannis, V.

This is a copy of the author's accepted version of a paper subsequently to be published in the proceedings of Intelligent Systems Conference 2017, London, UK, 07 to 09 Sep 2017, IEEE.

It is available online at:

https://dx.doi.org/10.1109/IntelliSys.2017.8324303

(C) 2017 IEEE . Personal use of this material is permitted. Permission from IEEE must be obtained for all other uses, in any current or future media, including reprinting/republishing this material for advertising or promotional purposes, creating new collective works, for resale or redistribution to servers or lists, or reuse of any copyrighted component of this work in other works.

The WestminsterResearch online digital archive at the University of Westminster aims to make the research output of the University available to a wider audience. Copyright and Moral Rights remain with the authors and/or copyright owners.

Whilst further distribution of specific materials from within this archive is forbidden, you may freely distribute the URL of WestminsterResearch: ((http://westminsterresearch.wmin.ac.uk/)).

In case of abuse or copyright appearing without permission e-mail repository@westminster.ac.uk 


\title{
Day ahead hourly Price Forecast in ISO New England Market using Neuro-Fuzzy Systems
}

\author{
Abeer Alshejari, Vassilis S. Kodogiannis \\ Faculty of Science and Technology \\ University of Westminster \\ London, United Kingdom \\ V.Kodogiannis@,westminster.ac.uk
}

\begin{abstract}
Accurate electricity price forecasting is an alarming challenge for market participants and managers owing to high volatility of the electricity prices. Price forecasting is also the most important management goal for market participants since it forms the basis of maximizing profits. These markets are usually organized in power pools and administrated by the independent system operator (ISO). The aim of this study is to examine the performance of asymmetric neuro-fuzzy network models for day-ahead electricity price forecasting in the ISO New England market. The implemented model has been developed with two alternative defuzzification models. The first model follows the Takagi-Sugeno-Kang scheme, while the second the traditional centre of average method. A clustering scheme is employed as a pre-processing technique to find out the initial set and adequate number of clusters and ultimately the number of rules in the network. Simulation results corresponding to the minimum and maximum electricity price indicate that the proposed network architectures could provide a considerable improvement for the forecasting accuracy compared to alternative learning-based schemes.
\end{abstract}

Keywords-Electricity price forecasting; neurofuzzy systems; neural networks; clustering; prediction

\section{INTRODUCTION}

During the past two decades we have seen widespread electricity sector liberalization and deregulation in all EU countries. With the introduction of restructuring into the electric power industry, the price of electricity has become the focal point of all activities in the power market [1]. Electricity price forecasting is a challenging task and is very important in competitive electricity market. The problem of electricity price forecasting is related yet distinct from that of electricity load forecasting. Although the load and the price are correlated, their relation is mainly non-linear. Power load is influenced by the factors such as non-storability of electricity, consumers' behavioral patterns, and seasonal changes in demand. Price, on the other hand, is affected by those aforesaid factors as well as additional aspects such as financial regulations, competitors' pricing, dynamic market factors, and various other macro and micro economic conditions. As a result, the price of electricity is a lot more volatile than the electricity load. Interestingly, when dynamic pricing strategies are introduced, prices become even more volatile, where the daily average price changes by up to $50 \%$ while other commodities may exhibits about $5 \%$ change [2].
Both market players and the regulators are concerned much about the price evolution. Market price prediction is vital information for the producers' production arrangement and bidding strategies. There are various methods adopted for the forecasting of future market price. One approach to predict the market behaviours is regression. The basic idea is the usage of historical prices, quantity and other information such as load forecast, and temperatures to predict the market-clearing price (MCPs). However, a simple linear regression model is unable to describe the complicated relation between load and electricity prices, because their relation is commonly dynamic and nonlinear [3]. Traditional ARMA models are able to find inherent rules of a time series by utilizing history data, but again they do not take into account the effect of other factors on electricity prices. Much work has been done on electricity price forecasting with the Auto Regressive Integrated Moving Average (ARIMA) method [4]. In particular, the ARIMA method has been extended to include error correction for the worse market conditions with high price volatility [5]. Techniques that are based on the wavelet transform and ARIMA model have been applied to Spanish power markets in order to improve the accuracy of price forecasting [6].

Due to their simplicity and flexibility, Neural Networks (NNs) have typically received much attention recently. While the majority of the studies refer to day-ahead predictions, the MLP network has been utilized in hour-ahead time framework [7]. In order to improve the accuracy of such methods for forecasting, different techniques have been combined with NNs. A feature selection technique, a relief algorithm, has been combined with NNs, while particle swarm optimization has been used for NN training [8-9]. K-Means clustering method has been used to find clusters for the number of neural networks. The wavelet and NN models have been fitted together for greater price forecasting accuracy [10]. RBF is another type of NNs that is utilized in the work of [11]. An RBF network includes a hidden and an output layer. This type of NNs is able to simulate complex relationships underlying the data and can adapt fast to possible changes of these relationships. Support Vector Machines (SVMs) provide a nonlinear mapping of the original data into high dimensional space. The boundaries of the new space are demarcated using linear function. SVMs provide a global solution to a problem unlike MLPs who can operate within local minima of their objective function. This fact has been also recognized in many research studies related to the load and price forecasting area 
[12]. Genetic algorithms, in combination with LSSVM (Least Square Support Vector Machine), have been proposed. It has been proven that the forecasting is more accurate than the original SVM forecasting [13].

One of the first applications of fuzzy logic to electricity price forecasting was performed by Hong [14], who utilize fuzzy c-means for classifying historical data into three clusters (peak, medium and off-peak), and then employ a recurrent network for forecasting. Another approach in price forecasting is the synergetic operation of Fuzzy Logic (FL) and NNs. This part of the literature can be further classified into two categories: Studies that utilize FL and NN in the same system (i.e. neuro-fuzzy systems like ANFIS) and the studies where FL and NN are separated forecasters that are combined into a two-part forecaster. An adaptive-network-based fuzzy inference system (ANFIS) has been investigated and results proved that such scheme is superior to MLP approaches [15].

In most of the price forecasting case studies, especially in the hourly price forecasting utilizing learning-based algorithms, only one model is built to forecast the 24 hourly prices of the next day. However, it is a rather difficult task to associate all the characteristics of 24 different hourly prices by one single model. Thus, the model may become under-fitting for some hourly prices; but at the same time, it may become over-fitting for some others, which leads to unsatisfactory results. An obvious disadvantage of this approach however is related to the high complexity of the network structure (i.e. a system with 24 output nodes) in terms of training time and performance. Alternatively, a recurrent structure could provide, in theory, similar characteristics, however in practice its performance would be deteriorated due to the feedback error accumulation. An alternative approach has been proposed in recent past [16] and it has been adopted also in this paper. The core of the proposed modular forecasting system is the 24 multi-input-single-output (MISO) modelling blocks. One of the flexibilities of the proposed module system is its possible use also for long-range forecasting schemes.

In this paper, neurofuzzy models are considered to compute the forecasted price in ISO New England market. The ISO New England market is coordinated by an independent system operator (ISO) (http://www.iso-ne.com). It has been observed that although the daily power demand curves having similar pattern, but the daily price curves are however volatile. Therefore, forecasting of Locational Marginal Prices (LMPs) become more important as it helps market participants not only to determine the bidding strategies of their generators, but also in risk management. In this work, the training/testing data set was created from the period 2006-2007. Both training and testing sets were classified into 24 time series, each one corresponding to a different hour of the day. More specifically, 600 data were allocated to training subset, while 123 data for the testing one. Two Asymmetric Gaussian Fuzzy Inference Neural Networks (AGFINN) utilizing a Takagi-Sugeno-Kang (TSK) and a centre of average defuzzification structures respectively have been considered as identification models for electricity price forecasting. Unlike the ANFIS system, AGFINN includes a clustering component which reduces the number of fuzzy rules, minimizing thus the "curse of dimensionality" problem. A fuzzy c-means (FCM) clustering algorithm is applied for the sample data in order to organize feature vectors into clusters such that points within a cluster are closer to each other than vectors belonging to different clusters. In the following result section, only results that correspond to hours with the maximum $(22: 00 \mathrm{~h})$ and minimum $(04: 00 \mathrm{~h})$ electricity prices are illustrated. The proposed modelling scheme is compared against ANFIS, AFLS, Wavelet network (WNN) and MLP NN forecasting schemes utilized for the same case study in order to evaluate its performance as an efficient prediction scheme.

\section{ASYMMETRIC NEUROFUZZY MODEL}

In this section, the proposed Asymmetric Gaussian Fuzzy Inference Neural Network (AGFINN) concept is presented as an alternative neurofuzzy modelling approach. Initially, AGFINN has been implemented as a MIMO neurofuzzy (NF) network which incorporates a clustering pre-processing stage. The architecture of the proposed scheme shown in Fig 1 includes also a FCM clustering scheme for structural / initialization purposes. In spite of the extensive use of the standard symmetric Gaussian membership functions, AGFINN utilizes an asymmetric function acting as input linguistic node. Since the asymmetric Gaussian membership function's variability and flexibility are higher than the traditional one, it can partition input space more effectively [17]. In this paper, AGFINN has been optimized through the gradient descent learning algorithm, while "centre average" (CA) defuzzifier has been used as defuzzification method. This technique is more efficient in terms of implementation compared to the traditional, for fuzzy logic systems, "centroid of area" approach [18].

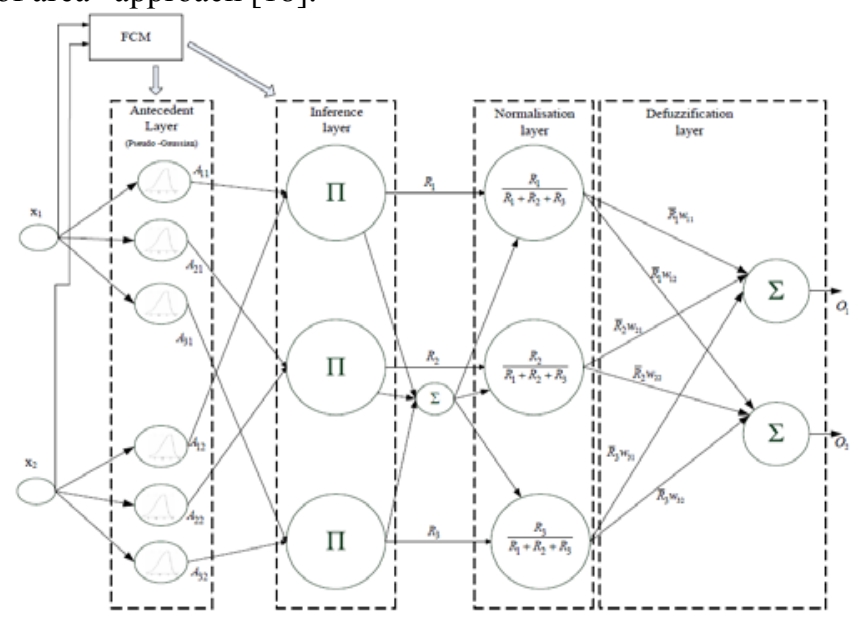

Fig. 1. Structure of AGFINN-CA system

Many neuro-fuzzy schemes are following the TSK defuzzification style, where only one output is enabled. ANFIS is a well-known representative of TSK-based neurofuzzy systems. However, ANFIS's main limitation is the exponential growth of rules subjected to the number of input variables. Generally, TSK-based models allow us to model nonlinear behaviour with relatively fast training speed. Thus, it would be interesting to investigate a TSK-based version for 
AGFINN and explore any possible improvement against ANFIS. Similarly to previous AGFINN-CA scheme, AGFINN-TSK has been built around five layers, utilising the same learning training algorithm. The architecture for AGFINN-TSK is shown in Fig 2.The first three layers $L_{1}, L_{2}$ and $\mathrm{L}_{3}$ correspond to IF part of fuzzy rules whereas layer $\mathrm{L}_{5}$ contains information about THEN part of these rules and perform the defuzzification task. In layer $\mathrm{L}_{4}$ a normalization process is performed for all rules derived from $\mathrm{L}_{3}$.

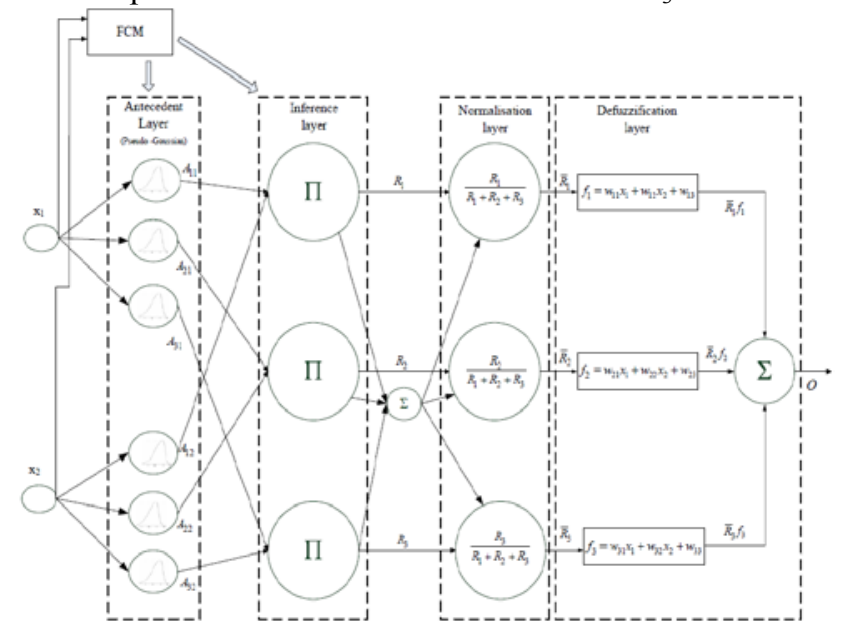

Fig. 2. Structure of AGFINN-TSK system

\section{A. FCM Clustering Algorithm}

Fuzzy c-means (FCM) clustering is the most prominent fuzzy unsupervised clustering algorithm which is based on minimizing an objective function that represents the distance from any given data point to a cluster centre weighted by that data point's membership value. Given $\mathrm{n}$ data patterns, $\mathrm{x}_{1}, \mathrm{x}_{2}, \ldots, \mathrm{x}_{\mathrm{n}}$ fuzzy clustering means partitioning the data patterns into c clusters which centred at $c_{i}$. The objective function for FCM is defined by

$$
\sum_{\mathrm{i}=1}^{\mathrm{c}} \sum_{\mathrm{j}=1}^{\mathrm{n}} \mu_{\mathrm{ij}}^{\mathrm{m}} \mathrm{d}_{\mathrm{ij}}^{2}, \quad 1 \leq \mathrm{i} \leq \mathrm{c}
$$

where $\mu_{\mathrm{ij}}$ is the degree of membership of object $\mathrm{j}$ in cluster $\mathrm{i}, \mathrm{m}$ is the weighting exponent varying in the range $[1, \infty]$ and $\mathrm{d}_{\mathrm{ij}}$ denotes the Euclidean distance between $\mathrm{x}_{\mathrm{j}}$ and $\mathrm{c}_{\mathrm{i}}$. The membership $\mu_{\mathrm{ij}}$ and the cluster centres $\mathrm{c}_{\mathrm{i}}$ are calculated by the following equations:

$$
\begin{gathered}
\mu_{\mathrm{ij}}=\left[\sum_{\mathrm{k}=1}^{\mathrm{c}}\left(\frac{\mathrm{d}_{\mathrm{ij}}}{\mathrm{d}_{\mathrm{kj}}}\right)^{(2 /(\mathrm{m}-1))}\right]^{-1}, 1 \leq \mathrm{i} \leq \mathrm{c}, \quad 1 \leq \mathrm{j} \leq \mathrm{n} \\
\mathrm{c}_{\mathrm{i}}=\frac{\sum_{\mathrm{j}=1}^{\mathrm{n}} \mu_{\mathrm{ij}}^{\mathrm{m}} \mathrm{x}_{\mathrm{j}}}{\sum_{\mathrm{j}=1}^{\mathrm{n}} \mu_{\mathrm{ij}}^{\mathrm{m}}}
\end{gathered}
$$

FCM clustering is an interactive procedure which updates $c_{i}$ using the last iteration's membership values. This algorithm moves objects between clusters until the objective function cannot be decreased further. The result is a set of clusters that are as compact and well-separated as possible. In the present study, cluster centres have been utilized as initial values for the centres of Gaussian membership functions, while the number of if-then rules for AGFINN modelling is equal to the number of clusters obtained through FCM clustering approach. The spread values for each membership function $\sigma_{i j}$ are initialized according to

$$
\sigma_{i j}=\left(\sum_{k=1}^{n} u_{i k}\left(x_{k j}-c_{i j}\right)^{2} / \sum_{k=1}^{n} u_{i k}\right)^{1 / 2}
$$

These values are calculated based on the matrix $\mathbf{U}$, where its elements correspond to the fuzzy memberships of input $\boldsymbol{x}_{\boldsymbol{k}}$ in the $i^{\text {th }}$ cluster and have centre values obtained again from FCM.

\section{B. Feed-forward analysis of AGFINN}

The clustering algorithm gives the fuzzy c-partition of the sample data. This result helps us to generate fuzzy rules base for AGFINN schemes. Fuzzy IF-THEN rules can be written in the following form:

$$
\operatorname{IF}\left(x_{1} \text { is } U_{1}^{i} \text { AND....AND } x_{q} \text { is } U_{q}^{i}\right) \operatorname{THEN}\left(y=w_{0}^{i}+w_{1}^{i} x_{1}+. .+w_{q}^{i} x_{q}\right)
$$

where $U$ are fuzzy sets defined based on c-partition of learning data $\mathrm{X}$. The structure of AGFINN schemes is explained below layer by layer:

- Layer 1: This layer is simply the input layer. Nodes in this layer pass on the input signals $x_{1}, x_{2}, \ldots, x_{n}$ to $\mathrm{L}_{2}$.

Layer 2: This layer is the fuzzification layer, and its nodes represent the fuzzy sets used in the antecedent parts of the fuzzy rules. A fuzzification node receives an input and determines the degree to which this input belongs to in the node's fuzzy set. The outputs of this layer are the values of the asymmetric Gaussian membership function (MF) for the input values.

$$
\begin{gathered}
A_{i j}=\exp \left(-\left(\frac{x_{i}-c_{i j}}{\sigma_{i j}^{l e f t}}\right)^{2}\right) U\left(x_{i} ;-\infty, c_{i j}\right)+ \\
\qquad \exp \left(-\left(\frac{x_{i}-c_{i j}}{\sigma_{i j}^{\text {right }}}\right)^{2}\right) U\left(x_{i} ; c_{i j}, \infty\right) \\
\text { where } U\left(x_{i} ; a, b\right)= \begin{cases}1 & \text { if } a \leq x_{i}<b \\
0 & \text { otherwise }\end{cases}
\end{gathered}
$$

From the above equation, it is obvious that the proposed MF utilizes two spreads, namely $\sigma_{\mathrm{ij}}^{\text {left }}$ and $\sigma_{\mathrm{ij}}^{\text {right }}$ respectively. Both of these parameters transform the traditional Gaussian function to a more asymmetric style which can provide greater flexibility from the original one. A schematic of the proposed MF is shown in Fig. 3. 


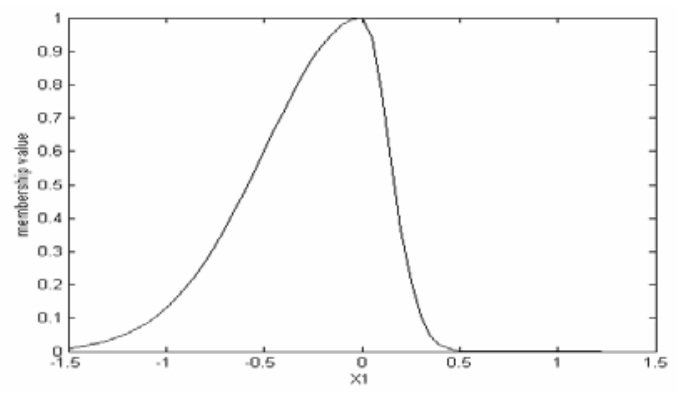

Fig. 3. Structure of AGFINN-TSK system

- Layer 3: This layer is the firing strength calculation layer. Since each fuzzy rule's antecedent part has AND connection operator, the firing strengths are calculated using the product $\mathrm{T}$-norm operator. In this case, the multiplication has been used, and the output of this layer has the following form:

$$
R_{j}=\prod_{i}^{n} A_{j i}\left(x_{i}\right)
$$

- Layer 4: This layer is the normalization layer. Each node in this layer calculates the normalized activation firing of each rule by:

$$
\bar{R}_{i}=\frac{R_{i}}{\sum_{j=1}^{c} R_{j}}
$$

- Layer 5: This layer is related to the defuzzification /output part of the AGFINN. Each node at this layer combines the output of each node in $\mathrm{L}_{4}$ by algebraic sum operation after being multiplied by the output weight value $f_{j}$ or $w_{i j}$ :

$$
O_{i}=\sum_{j=1}^{c} w_{i j} \bar{R}_{j} \quad(C A) \quad \text { or } \quad O=\sum_{j=1}^{c} f_{j} \bar{R}_{j} \quad(T S K)
$$

where $f_{j}=w_{j 1} x_{1}+\ldots+w_{j n} x_{n}+w_{j(n+1)} \quad$ represent the "consequent parameters" of the TSK-style defuzzification scheme.

The learning algorithm of AGFINN involves the use of the gradient descent (GD) method to optimize the various network parameters. During, the backward "training" passes, the error signals are calculating from the output layer backward to the premise (i.e. membership) layers, and parameters at both defuzzification and fuzzification sections are fine-tuned.

\section{RESULTS \& DISCUSSION}

Electricity price is a nonlinear function of many input variables, including their past values as well as past and forecasted values of any exogenous variables such as load consumption. To deal with this fact, three different models have been considered for this study, in order to extract safe conclusions about the forecasting approach that needs to be followed for the specific dataset. In the majority of forecasting problems, historical values of the parameter under study have always been considered as input candidates. In electricity price analysis, the most influential external variable is considered to be the load. In this study, we assume that next day's forecasted load is available. There is an analogy between price and load values. While the load level rises, a constant increase of price is also observed.

\section{A. Model A}

The objective of this model is to examine a simple configuration, used by various researchers, where electricity prices at previous days and hours, as well as forecasted (for the targeted hour/day) load demand are utilized as input variables. Thus, for electricity price modelling for a specific hour (i) and day (j), the following five input variables have been considered:

\section{Target:}

- $\quad$ Price $(i, j)$ : electricity price at the $i^{\text {th }}$ hour on the $(j)^{\text {th }}$ day,

\section{Inputs:}

- $\quad$ Price $(i, j-1)$ : price at the $i^{\text {th }}$ hour on the $(j-1)^{\text {th }}$ day,

- Price $(i, j-2):$ price at the $i^{\text {th }}$ hour on the $(j-2)^{\text {th }}$ day,

- Price $(i-1, j-1)$ : price at the $(i-1)^{\text {th }}$ hour on the $(j-$ 1) ${ }^{\text {th }}$ day,

- Price $(i-2, j-1)$ : price at the $(i-2)^{\text {th }}$ hour on the $(j-$ 1) $)^{\text {th }}$ day,

- $\operatorname{Load}(i, j)$ : electricity load at the $i^{\text {th }}$ hour on the $j^{\text {th }}$ day,

Based on this configuration, AGFINN models have been involved in forecasting the maximum (22h) and minimum (04h) price respectively. Best results were produced by including 20 fuzzy rules for the case of $22 \mathrm{~h}$, while 15 rules were adequate for the case of $04 \mathrm{~h}$. Although the classic gradient method utilized as a learning scheme, the training time was completed in less than 1000 epochs, much faster from the equivalent time used to train the MLP neural network. The performance of the forecasting model was determined by the root mean squared error (RMSE), the Mean absolute percentage error (MAPE) (\%) and finally and the standard error of prediction (SEP).

TABLE I

PERFORMANCE INDICES

\begin{tabular}{|l|c|c|c|c|}
\hline \multicolumn{1}{|c|}{$\begin{array}{c}\text { Statistical index for AGFINN } \\
\text { (Model A) }\end{array}$} & \multicolumn{2}{|c|}{$\begin{array}{c}\text { Testing Datasets } \\
\text { (TSK) }\end{array}$} & \multicolumn{2}{c|}{$\begin{array}{c}\text { Testing Data sets } \\
\text { (CA) }\end{array}$} \\
\cline { 2 - 5 } & $\mathbf{2 2 h}$ & $\mathbf{0 4 h}$ & $\mathbf{2 2 h}$ & $\mathbf{0 4 h}$ \\
\hline Root mean square error (RMSE) & 7.9028 & 3.3783 & 8.110 & 4.0542 \\
\hline $\begin{array}{l}\text { Mean absolute percentage error (MAPE) } \\
\text { (\%) }\end{array}$ & 5.0385 & 7.0090 & 4.9384 & 7.7738 \\
\hline Standard error of prediction (SEP) (\%) & 6.5310 & 7.5578 & 6.7022 & 9.0700 \\
\hline
\end{tabular}


The complete results for the hours with minimum and maximum electricity price are illustrated in Table I. The RMSE index is calculated between the desired and output values and then averaged across all data and it can be used as an estimation of the goodness of fit of the models. It can also provide information about how consistent the model would be in the long run. The MAPE term is the average absolute percent error for each time period or forecast minus actual, divided by actual. The SEP index is determined as the relative deviation of the mean prediction values and it has the advantage of being independent on the magnitude of the measurements. Based on these indices, the AGFINN scheme achieved a very good performance, especially for the case of maximum price. In order to evaluate the goodness of the current performance of the proposed AGFINN schemes, a comparison against NN, WNN and neurofuzzy models that have been employed for the specific datasets has been carried out. Table II provides a summary of those statistical performances. More specifically, AGFINN schemes have been compared against a multilayer perceptron (MLP), wavelet NN and neurofuzzy (NF) ANFIS and AFLS systems.

TABLE II

PERFORMANCE INDICES - COMPARISON

\begin{tabular}{|c|c|c|c|c|}
\hline Statistical index (22h) & AFLS & ANFIS & WNN & MLP \\
\hline Root mean square error (RMSE) & 8.6060 & 10.3569 & 9.2798 & 12.8880 \\
\hline 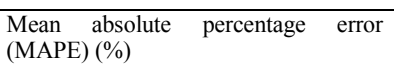 & 5.4587 & 6.3012 & 6.1393 & 7.4165 \\
\hline Standard error of prediction (SEP) (\%) & 7.1122 & 8.5591 & 7.6690 & 10.6508 \\
\hline Statistical index (04h) & AFLS & ANFIS & $\overline{\text { WNN }}$ & $\overline{\text { MLP }}$ \\
\hline Root mean square error (RMSE) & 4.6194 & 7.5882 & 8.7796 & 10.4905 \\
\hline $\begin{array}{llll}\text { Mean absolute } & \text { percentage } & \text { error } \\
(\text { MAPE) }(\%) & & \end{array}$ & 9.4320 & 13.6217 & 10.6214 & 21.3592 \\
\hline Standard error of prediction (SEP) (\%) & 10.3345 & 16.9763 & 19.6418 & 23.4693 \\
\hline
\end{tabular}

The "Adaptive Fuzzy Logic System" (AFLS) model is an advanced MIMO NF systems which includes a prototype defuzzification scheme, while differs from conventional fuzzy rule-table approaches that utilize the "look-up table" concept [19]. The AFLS scheme does not follow TSK's architecture, as the number of memberships for each input variable is directly associated to the number of rules, hence, the "curse of dimensionality" problem is significantly reduced. The fuzzification component in AFLS is similar to AGFINN, with the exception of the FCM clustering step as well as the absence of asymmetric MFs. For this specific case study, 20 fuzzy rules for the case of $22 \mathrm{~h}$, and 15 rules for the case of $04 \mathrm{~h}$ were used as a final configuration. Results shown at Table II reveal that AFLS could be considered as the closest to AGFINN-CA in terms of performance. An MLP network was also constructed for this case study, using the same input vector. After a few trials, utilizing different internal structures, a NN was implemented with two hidden layers (with 20 and 8 nodes respectively). Although AGFINN, AFLS and MLP share the same learning training algorithm, the different "philosophy" in building the neurofuzzy architecture, allowed those systems to achieve a superior performance. An ANFIS NF model has been constructed, utilising 32 fuzzy rules. As the number of MFs in AGFINNs is equal to the numbers of rules, the proposed architecture has advantages over the classic ANFIS model. An interesting finding from this simulation is related to the performance of $\mathrm{WNN}$, which outperformed ANFIS for the case of $22 \mathrm{~h}$. More specifically, 20 Morlet wavelet functions were utilised in the construction of WNN [20].

\section{B. Model B}

Research has indicated that current hour electricity price shows a high correlation with those of hour h-24 and h-168, a fact that indicates daily and weekly periodicity. The objective of this model is to investigate this issue. No exogenous input variables are considered in this specific case study. Thus, for electricity price modelling for a specific hour (i) and day (j), the following six input variables have been considered:

\section{Target:}

- Price $(i, j)$ : electricity price at the $i^{\text {th }}$ hour on the $(j)^{\text {th }}$ day,

\section{Inputs:}

- Price $(i, j-1)$ : price at the $i^{t^{t h}}$ hour on the $(j-1)^{\text {th }}$ day,

- $\quad$ Price $(i, j-2)$ : price at the $i^{\text {th }}$ hour on the $(j-2)^{\text {th }}$ day,

- Price $(i, j-3)$ : price at the $i^{\text {th }}$ hour on the $(j-3)^{\text {th }}$ day,

- $\quad$ Price $(i, j-7)$ : price at the $i^{i^{\text {th }}}$ hour on the $(j-7)^{\text {th }}$ day,

- Price $(i-1, j-1)$ : price at the $(i-1)^{\text {th }}$ hour on the $(j-1)^{\text {th }}$ day,

- Price $(i-2, j-1)$ : price at the $(i-2)^{\text {th }}$ hour on the $(j-1)^{\text {th }}$ day,

The complete results for the hours with minimum and maximum electricity price, for the AGFINN case are illustrated in Table III. The information related to weekly periodicity indeed resulted in an improved forecasting performance compared to Model A. Best results were produced by including 25 fuzzy rules for the case of $22 \mathrm{~h}$, while 20 rules were adequate for the case of $04 \mathrm{~h}$.

TABLE III

PERFORMANCE INDICES

\begin{tabular}{|l|c|c|c|c|}
\hline \multicolumn{1}{|c|}{$\begin{array}{c}\text { Statistical index for AGFINN } \\
\text { (Model B) }\end{array}$} & \multicolumn{2}{|c|}{$\begin{array}{c}\text { Testing Datasets } \\
\text { (TSK) }\end{array}$} & \multicolumn{2}{c|}{$\begin{array}{c}\text { Testing Data sets } \\
\text { (CA) }\end{array}$} \\
\cline { 2 - 5 } & $\mathbf{2 2 h}$ & $\mathbf{0 4 h}$ & $\mathbf{2 2 h}$ & $\mathbf{0 4 h}$ \\
\hline Root mean square error (RMSE) & 7.3320 & 3.3089 & 7.6515 & 3.7748 \\
\hline $\begin{array}{l}\text { Mean absolute percentage error (MAPE) } \\
\text { (\%) }\end{array}$ & 4.5763 & 6.2654 & 4.5552 & 7.5610 \\
\hline Standard error of prediction (SEP) (\%) & 6.0593 & 7.4027 & 6.3233 & 8.4450 \\
\hline
\end{tabular}

All statistical performance indices were improved at this case study, compared to Model A. This was due to the expansion of 
input variables vector by adding additional past electricity prices on the same hour. In fact, the assumption that electricity prices "contain" a periodicity effect was verified by this simulation. Results shown at Table IV illustrate results from alternative methods. For this case study, an AFLS model was constructed with 25 rules for the case of $22 \mathrm{~h}$, while 20 rules were used for the case of 04h. The MLP NN retained the same network configuration, while under these conditions, ANFIS performed satisfactory, its performance however was achieved with a high computational cost, by utilizing two membership functions for each input variables and 64 fuzzy rules.

TABLE IV PERFORMANCE INDICES - COMPARISON

\begin{tabular}{|c|c|c|c|c|}
\hline Statistical index (22h) & AFLS & ANFIS & WNN & MLP \\
\hline Root mean square error (RMSE) & 7.9278 & 9.8380 & 8.5303 & 11.6525 \\
\hline $\begin{array}{llll}\text { Mean } & \text { absolute } & \text { percentage } & \text { error } \\
(\text { MAPE) }(\%) & & \end{array}$ & 4.8062 & 6.1757 & 5.4173 & 6.9578 \\
\hline Standard error of prediction (SEP) (\%) & 6.5517 & 8.1302 & 7.0496 & 9.6298 \\
\hline Statistical index (04h) & AFLS & ANFIS & WNN & MLP \\
\hline Root mean square error (RMSE) & 4.5427 & 6.0034 & 6.755 & 8.7796 \\
\hline $\begin{array}{llll}\text { Mean } & \text { absolute } & \text { percentage } & \text { error } \\
(\mathrm{MAPE})(\%) & & \end{array}$ & 9.5050 & 12.3199 & 12.140 & 10.6214 \\
\hline Standard error of prediction (SEP) (\%) & 10.163 & 13.4307 & 14.121 & 19.6418 \\
\hline
\end{tabular}

ANFIS is a classic representative of TSK-based neuro-fuzzy systems. Generally, in this type of models, an input space is divided into $K_{1} \times K_{2} \times \ldots \times K_{n}$ fuzzy subspaces, where $K_{i}$, $i=1,2, \ldots, n$ is the number of fuzzy subsets for the $i^{\text {th }}$ input variable. As one fuzzy rule is normally assigned for each one of these subspaces, their main drawback is that the number of fuzzy rules increases exponentially with respect to the number of inputs $n$. Results from this case study, indicate that may ANFIS scheme is not a suitable identification model for cases with large input vectors. The WNN however, reveal a remarkable robustness against ANFIS, resulting in a similar performance with only 20 wavelet MFs.

\section{Model C}

The objective of this model is to expand Model B, by adding the exogenous input of the forecasted electricity load. Thus, for electricity price modelling for a specific hour (i) and day (j), the following seven input variables have been considered:

Target:

Inputs:

- Price $(i, j):$ electricity price at the $i^{\text {th }}$ hour on the $(j)^{\text {th }} d a y$,

- Price $(i, j-1)$ : price at the $i^{\text {th }}$ hour on the $(j-1)^{\text {th }}$ day,

- Price $(i, j-2)$ : price at the $i^{\text {th }}$ hour on the $(j-2)^{\text {th }}$ day,

- Price $(i, j-3)$ : price at the $i^{\text {th }}$ hour on the $(j-3)^{\text {th }}$ day,

- Price $(i, j-7)$ : price at the $i^{\text {th }}$ hour on the $(j-7)^{\text {th }}$ day,

- Price $(i-1, j-1)$ : price at the $(i-1)^{\text {th }}$ hour on the $(j-$ $1)^{\text {th }}$ day,
- Price $(i-2, j-1)$ : price at the $(i-2)^{\text {th }}$ hour on the $(j-$ $1)^{\text {th }}$ day,

- Load $(i, j)$ : electricity load at the $i^{\text {th }}$ hour on the $j^{\text {th }}$ day,

The complete results for the hours with minimum and maximum electricity price, for the AGFINN case are illustrated in Table V.

TABLE V

PERFORMANCE INDICES

\begin{tabular}{|l|c|c|c|c|}
\hline \multicolumn{1}{|c|}{$\begin{array}{c}\text { Statistical index for AGFINN } \\
\text { (Model C) }\end{array}$} & \multicolumn{2}{c|}{$\begin{array}{c}\text { Testing Data sets } \\
\text { (TSK) }\end{array}$} & \multicolumn{2}{c|}{$\begin{array}{c}\text { Testing Data sets } \\
\text { (CA) }\end{array}$} \\
\cline { 2 - 5 } & $\mathbf{2 2 h}$ & $\mathbf{0 4 h}$ & $\mathbf{2 2 h}$ & $\mathbf{0 4 h}$ \\
\hline Root mean square error (RMSE) & 6.8514 & 2.9988 & 7.5032 & 3.5844 \\
\hline $\begin{array}{l}\text { Mean absolute percentage error (MAPE) } \\
\text { (\%) }\end{array}$ & 4.2418 & 5.4832 & 5.0097 & 6.9741 \\
\hline Standard error of prediction (SEP) (\%) & 5.6621 & 6.7089 & 6.2007 & 8.0189 \\
\hline
\end{tabular}

The information related to weekly periodicity as well as the exogenous load parameter resulted in an improved forecasting performance compared to previous case studies. Best AGFINN results were produced by including 25 fuzzy rules for the case of $22 \mathrm{~h}$, while 20 rules were adequate for the case of $04 \mathrm{~h}$. All statistical performance indices were improved at this case study, compared to Models A and B. This was due to the expansion of input variables vector by adding additional past electricity prices on the same hour. In fact, the assumption that electricity prices "contain" a periodicity effect was verified also by this simulation.

TABLE VI.

PERFORMANCE INDICES - COMPARISON

\begin{tabular}{|l|c|c|c|c|}
\hline Statistical index (22h) & AFLS & ANFIS & WNN & MLP \\
\hline Root mean square error (RMSE) & 7.7340 & 9.1584 & 8.0368 & 11.4835 \\
\hline $\begin{array}{l}\text { Mean absolute percentage error (MAPE) } \\
\text { \%) }\end{array}$ & 4.4775 & 5.3308 & 4.9288 & 6.0115 \\
\hline Standard error of prediction (SEP) (\%) & 6.3915 & 7.5686 & 6.6417 & 9.4901 \\
\hline & \multicolumn{4}{|l|}{} \\
\hline Statistical index (04h) & AFLS & ANFIS & WNN & MLP \\
\hline Root mean square error (RMSE) & 4.3667 & 5.4409 & 5.4184 & 8.0055 \\
\hline $\begin{array}{l}\text { Mean absolute percentage error (MAPE) } \\
\text { (\%) }\end{array}$ & 7.6772 & 8.3168 & 10.3565 & 16.5878 \\
\hline Standard error of prediction (SEP) (\%) & 9.7692 & 12.1724 & 11.622 & 17.9098 \\
\hline
\end{tabular}

Similarly, to previous case study, AFLS, ANFIS, WNN and MLP NN have been applied to this specific case study and their performances are presented at Table VI. ANFIS's performance was achieved however with a huge computational cost, by utilizing 128 fuzzy rules. It seems the only comparable method to AGFINN is the AFLS scheme. Even WNN approach outperformed ANFIS in this specific case study, revealing ANFIS's deficiencies. 
Figures 4 and 5 illustrate the testing performances for minimum and maximum electricity price forecasting using Model C, using AGFINN-TSK scheme.

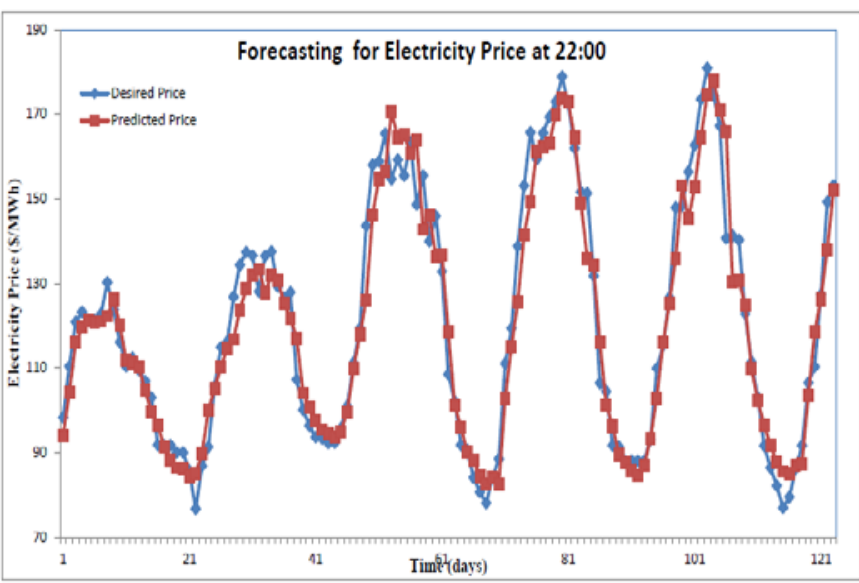

Fig. 4. Forecasting for Electricity Price at 22:00, (AGFINN-Model C)

Comparison of the proposed two AGFINN-based models indicates that TSK version is far superior to model utilising CA defuzzification scheme. AFGINN-TSK has also advantages over AFLS as well as TSK-based schemes like ANFIS.

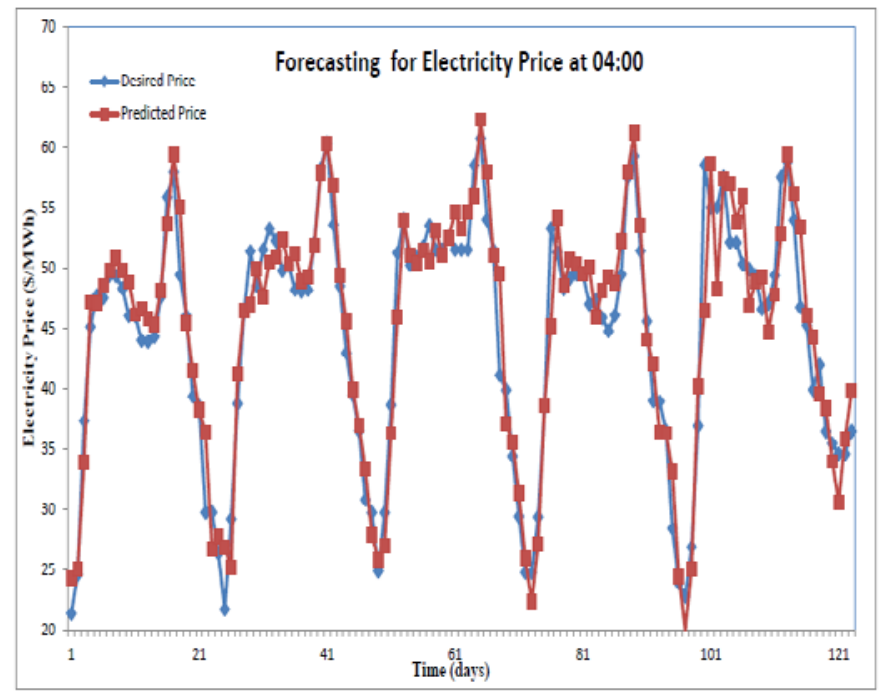

Fig. 5. Forecasting for Electricity Price at 04:00, (AGFINN-Model C)

\section{CONCLUSIONS}

An approach is proposed in this paper for short-term electricity prices forecasting, based on an asymmetric neuro-fuzzy identification model. The application of the proposed approach to electricity prices forecasting on the New England market is novel in terms of network architecture and forecasting performance. The effectiveness of this approach has been thoroughly assessed by comparing it with alternative neural or neurofuzzy techniques, via three case studies. Future research includes the incorporation in the modelling process additional exogenous parameters, as well as the adoption of recursive least squares algorithm for the optimization of the consequent component at AGFINN-TSK model.

\section{REFERENCES}

1. Conejo A.J., Contreras J., Espínola R., Plazas M.A., "Forecasting electricity prices for a day-ahead pool-based electric energy market”, Int. J Forecasting 21(3), 435-462, 2005.

2. Ziel F., Steinert R., Husmann S. "Efficient modeling and forecasting of electricity spot prices", Energy Econ 47, 98-111, 2015.

3. Cuaresma C.J., Hlouskova J., Kossmeier S., Obersteiner M., "Forecasting electricity spot-prices using linear univariate timeseries models", Applied Energy 77, 87-106, 2004.

4. Zhou M., Yan Z., Ni Y.X., Li G. and Nie Y. "Electricity Price Forecasting with Confidence-interval Estimation through an Extended ARIMA Approach", IEE Proc.- Gener. Tansm. Distrib. 153(2), 187-195, 2006.

5. Contreras J., Espinola R., Nogales F.J., Conejo A.J., “ARIMA Models to Predict Next-Day Electricity Prices", Power Engineering Review, IEEE 22(9), 57 - 87, 2002.

6. Conejo A.J., Plazas M.A., Espinola R., Molina A.B., "DayAhead Electricity Price Forecasting Using the Wavelet Transform and ARIMA Models", IEEE Transactions on Power Systems 20(2), 1035 - 1042, 2005.

7. Anbazhagan S., Kumarappan N. "Day-ahead deregulated electricity market price classification using neural network input featured by DCT", Int. J. Electr. Power Energy Syst. 37, 103109, 2012.

8. Amjady N., Daraeepour A. "Day-ahead Electricity Price Forecasting Using the Relief Algorithm and Neural Networks", 5th International Conference on European Electricity Market, Lisbon, 1-7, 2008.

9. Srinivasan D., Yong F.C., Liew A.C., "Electricity Price Forecasting Using Evolved Neural Networks", Inter. Conference on Intelligent Systems Applications to Power Systems, Niigata, $1-7,2007$.

10. Xian Z., Xi-Fan W., Fang-Hua C., Bin Y., and Hao-Yong C., "Short- Term Electricity Price Forecasting Based on PeriodDecoupled Price Sequence", Proceedings of the CSEE, 25(15), 1-6, 2005.

11. Yun Z., Quan Z., Caixin S., Shaolan L., Yuming L., Yang S., "RBF neural network and ANFIS-based short-term load forecasting approach in real-time price environment", IEEE Trans Power Systems 23(3), 853-858, 2008.

12. Mohamed A., El-Hawary M.E., "Mid-term electricity price forecasting using SVM", 2016 IEEE Canadian Conference on Electrical and Computer Engineering, 2016, No. 7726765.

13. Mahjoob M.J., Abdollahzade M., and Zarringhalam R., "GA based Optimized LSSVM Forecasting of Short Term Electricity Price in Competitive Power Markets", 3rd IEEE Conference on Industrial Electronics and Applications, Singapore, 73 - 78, 2008.

14. Hong Y.-Y., Hsiao C.-Y. "Locational marginal price forecasting in deregulated electricity markets using artificial intelligence", IEE Proceedings: Generation, Transmission and Distribution, 149(5), 621-626, 2002.

15. Catalao J.P., Pousinho H.M., Mendes V.M., "Hybrid waveletPSO-ANFIS approach for short-term electricity prices forecasting”, EEE Trans Power Systems, 26(1), 137-144, 2011. 
16. Kodogiannis V.S., Amina M., Petrounias I., “A clustering-based fuzzy-wavelet neural network model for short-term load forecasting”, Int. Journal of Neural Systems, 23(5), 2013.

17. Rojas I., Pomares H., Fernandez F.J., "A new methodology to obtain fuzzy systems autonomously from training data", IEEE conf. Fuzzy System, 1, 527-532, 1999.

18. Mendel J.M., "Fuzzy Logic Systems for Engineering: A Tutorial", Proceeding of the IEEE 83(3), 345-377, 1995

19. Kodogiannis V.S., Pachidis T., Kontogianni E., "An intelligent based decision support system for the detection of meat spoilage”, Eng. Appl. of Artificial Intelligence, 34, 23-36, 2014.

20. Amina M., Panagou E.Z., Kodogiannis V.S., Nychas G.J-E., "Wavelet neural networks for modelling high pressure inactivation kinetics of Listeria monocytogenes in UHT whole milk", Chemometrics and intelligent laboratory systems 103 (2), 170-183, 2010. 